199 BARICITINIB IN PATIENTS WITH SYSTEMIC LUPUS ERYTHEMATOSUS: RESULTS FROM A PHASE 2, RANDOMIZED, DOUBLE-BLIND, PLACEBO-CONTROLLED STUDY

${ }^{1}$ Maria E Silk*, ${ }^{2}$ Daniel J Wallace, ${ }^{3}$ Richard A Furie, ${ }^{4}$ Yoshiya Tanaka, ${ }^{5}$ Kenneth C Kalunian, ${ }^{6}$ Marta Mosca, ${ }^{7}$ Michelle Petri, ${ }^{8}$ Thomas Dörner, ${ }^{9}$ Mario H Cardiel, ${ }^{10}$ lan N Bruce, ${ }^{1}$ Elisa Gomez, ${ }^{1}$ Amy M DeLozier, 'Jonathan M Janes, 'Matthew D Linnik, 'Stephanie de Bono, ${ }^{1}$ Robert W Hoffman. ${ }^{1}$ Eli Lilly and Company; ${ }^{2}$ Cedars-Sinai Medical Center/David Geffen School of Medicine University of California Los Angeles; ${ }^{3}$ Zucker School of Medicine at Hofstra/Northwell; ${ }^{4}$ University of Occupational and Environmental Health, Japan; ${ }^{5}$ University of California at San Diego School of Medicine; ${ }^{6}$ University of Pisa; ${ }^{7}$ Johns Hopkins University School of Medicine; ${ }^{8}$ Charite Universitätsmedizin Berlin and Deutsches Rheuma-Forschungszentrum (DRFZ); ${ }^{9}$ Centro de Investigación Clínica de Morelia SC; ${ }^{10}$ The University of Manchester, Arthritis Research UK Centre for Epidemiology, Centre for Musculoskeletal Research, Faculty of Biology Medicine and Health

\subsection{6/lupus-2019-Ism.199}

Background Baricitinib (Bari), an oral selective inhibitor of Janus kinase (JAK)1 and JAK2, has been approved for the treatment of rheumatoid arthritis (RA) in over 50 countries including the United States, European countries, and Japan. The purpose of this study was to report results from a 24 week global, Phase 2, double-blind, placebo (PBO)-controlled study of Bari in patients with systemic lupus erythematosus (SLE) receiving standard therapy.

Methods Patients with SLE (positive antinuclear antibody [ANA] or anti-double stranded DNA [anti-dsDNA], clinical

Abstract 199 Table 1 Efficacy and safety outcomes of patients with systemic lupus erythematosus in a phase 2 study of baricitinib

\begin{tabular}{llll}
\hline & $\begin{array}{l}\text { PBO } \\
(\mathbf{n}=105)\end{array}$ & $\begin{array}{l}\text { Bari } 2 \mathrm{mg} \\
(\mathbf{n}=105)\end{array}$ & $\begin{array}{l}\text { Bari } 4 \mathrm{mg} \\
(\mathbf{n}=104)\end{array}$ \\
\hline Efficacy measure & Week 24 & & \\
Resolution of arthritis or rash (SLEDAI-2K) & $56(53.3)$ & $61(58.1)$ & $70(67.3)^{*}$ \\
SRI-4 & $50(47.6)$ & $54(51.4)$ & $67(64.4)^{*}$ \\
Flare (SFI, any severity) & $54(51.4)$ & $45(42.9)$ & $34(32.7)^{*}$ \\
Flare (SFI, severe) & $12(11.4)$ & $10(9.5)$ & $6(5.8)$ \\
LLDAS & $27(25.7)$ & $35(33.3)$ & $40(38.5)^{*}$ \\
$\Delta$ Tender joint count & -5.59 & -6.50 & $-6.86^{*}$ \\
$\Delta$ Swollen joint count & -4.60 & -4.12 & -4.76 \\
$\Delta$ Physician's Global Assessment & -26.3 & -25.9 & $-32.2^{*}$ \\
$\Delta$ Complement C3, g/L & 0 & 0 & -0.02 \\
$\Delta$ Complement C4, g/L & 0.01 & -0.01 & -0.01 \\
Safety measure & Weeks 0-24\$ & \\
TEAEs & $68(64.8)$ & $75(71.4)$ & $76(73.1)$ \\
SAEs & $5(4.8)$ & $11(10.5)$ & $10(9.6)$ \\
Serious infections & $1(1.0)$ & $2(1.9)$ & $6(5.8)$ \\
Deep vein thrombosis & 0 & 0 & $1(1.0)$ \\
\hline
\end{tabular}

$\Delta=$ least squares mean change from baseline; Bari=baricitinib; LLDAS=Lupus Low Disease Activity State; $n=$ number of patients in the analysis population; $n=$ number of patients in the specified category; $\mathrm{PBO}=$ placebo; $\mathrm{SAEs}=$ serious adverse events; $\mathrm{SFI}=$ Safety of Estrogens in Lupus Erythematosus National Assessment [SELENA]-Systemic Lupus Erythematosus Disease Activity Index [SLEDAI] Flare Index; SLEDAI-2K=Systemic Lupus Erythematosus Disease Activity Index 2000; SRI-4=Systemic Lupus Erythematosus Responder Index-4; TEAEs=treatment emergent adverse events.

Data are $n(\%)$ patients, unless otherwise indicated.

${ }^{*} \mathrm{p} \leq 0.05$ vs placebo.

Includes up to 30 days post-treatment.

Wallace et al. Baricitinib in patients with systemic lupus erythematosus: results from a phase 2, randomized, double-blind, placebo-controlled study [abstract].

Arthritis Rheumatol. 2018; 70 (suppl 10).

Wallace et al. Baricitinib for systemic lupus erythematosus: a double-blind, randomised, placebo-controlled, phase 2 trial. Lancet. 2018; 392: 222-31.
SLE Disease Activity Index 2000 [SLEDAI-2K] 4, arthritis or rash required) receiving stable background SLE therapy were randomized 1:1:1 to $\mathrm{PBO}$ or Bari (2- or $4 \mathrm{mg}$ ) once-daily. The primary endpoint was resolution of arthritis or rash as defined by the SLEDAI-2K at Week 24.

Results Of 314 patients randomized, 79\%, 82\%, and $83 \%$ completed 24 weeks of treatment in the PBO, Bari $2 \mathrm{mg}$, and Bari $4 \mathrm{mg}$ groups, respectively. At Week 24, a significantly greater proportion of patients in the Bari $4 \mathrm{mg}$ group compared to PBO achieved resolution of arthritis or rash $(67 \%$ vs $53 \%, \mathrm{p}<0.05)$ and SLE Responder Index (SRI)-4 response $(64 \%$ vs $48 \%, \mathrm{p}<0.05)$. At Week 24, the proportions of patients achieving flare reduction (Safety of Estrogens in Lupus Erythematosus National Assessment [SELENA]-SLEDAI Flare Index [SFI]), Lupus Low Disease Activity State (LLDAS), Physicians Global Assessment, and tender joint count change from baseline were also significantly improved for Bari $4 \mathrm{mg}$ compared to PBO (Table). No statistically significant differences were observed between Bari $2 \mathrm{mg}$ and $\mathrm{PBO}$ in any of the above endpoints. There were no significant changes in antidsDNA or complement in patients treated with Bari. Rates of adverse events leading to treatment discontinuation and serious adverse events (SAEs) were higher for both Bari dose groups compared to PBO. There were no deaths, malignancies, major adverse cardiovascular events, tuberculosis, or serious herpes zoster infections; $1 \mathrm{SAE}$ of deep vein thrombosis was reported in a patient with risk factors (Bari $4 \mathrm{mg}$ group).

Conclusions In patients with SLE receiving standard background therapy, once-daily oral Bari $4 \mathrm{mg}$ was associated with significant clinical improvements compared to $\mathrm{PBO}$ and an acceptable benefit/risk profile. These findings support further study of Bari as a potential therapy for patients with SLE. Funding Source(s): This study was funded by Eli Lilly and Company.

\section{EFFICACY AND SAFETY OF BELIMUMAB IN PATIENTS OF BLACK RACE WITH SYSTEMIC LUPUS ERYTHEMATOSUS: RESULTS FROM THE EMBRACE STUDY}

'David D'Cruz, ${ }^{2}$ Kathleen Maksimowicz-McKinnon, ${ }^{3} \mathrm{Sim}$ Oates*, ${ }^{4}$ Mittermayer Barreto Santiago, ${ }^{5}$ Damon Bass, ${ }^{6}$ Susan Burriss, ${ }^{7}$ Jennifer Gilbride, ${ }^{8}$ James Groark, ${ }^{6}$ Michelle Miller, ${ }^{9}$ Beulah Ji. ' Louise Coote Lupus Unit, Guys Hospital, London, UK; ${ }^{2}$ Henry Ford Hospital, Wayne State University, Detroit, MI, USA; ${ }^{3}$ Division of Rheumatology and Immunology, Medical University of South Carolina; ${ }^{4}$ Bahia School of Medicine and Public Health; ${ }^{5}$ GlaxoSmithKline, Immuno-inflammation and Future Pipeline, Collegeville, $P A$, USA; ${ }^{6}$ GSK, Collegeville, PA, USA; ${ }^{7}$ GSK, Stevenage, Hertfordshire, UK; ${ }^{8}$ GlaxoSmithKline; ${ }^{9} G S K$, Uxbridge, Middlesex, UK

\subsection{6/lupus-2019-Ism.200}

Background Black patients have an increased prevalence and severity of systemic lupus erythematosus (SLE), alongside higher mortality rates. The efficacy and safety of intravenous (IV) belimumab has been demonstrated in several Phase $2 / 3$ studies; however, the small number of black patients within these trials, and the conflicting results, have limited conclusions regarding efficacy in this population. The objective of this study was to specifically assess the efficacy and safety of IV belimumab plus standard of care (SoC) in black patients with active, auto antibody-positive SLE.

Methods EMBRACE (NCT01632241) is a randomized, multicenter, double-blind, placebo-controlled trial in patients of self identified black race, aged 18 years, with active SLE at 